\title{
A Simple Approach for Determining Core-loss of Magnetic Materials \\ C.F.FOO,D.M.ZHANG,X.LI
}

School of Electrical and Electronic Engineering,

Nanyang Technological University,

Nanyang Avenue, Singapore 639798

\begin{abstract}
In the design of high frequency high efficiency dc power supply, core losses in magnetic components becomes an important design consideration. A simple in-house experimental setup consisting of an impedance analyzer and a $\mathrm{PC}$ computer for determining core loss of $\mathrm{Mn}-\mathrm{Zn}$ magnetic materials under sinusoidal excitation is proposed here. The power requirement for this measurement is small. The method using compensating technique provides reasonably accurate result as the determination of core loss is not significantly affected by the parasitic of the measuring apparatus especially when measurement is done at high frequency.
\end{abstract}

\section{INTRODUCTION}

In the design of high-frequency high-efficiency dc power supplies, core loss in the magnetic components is important design consideration. Information of magnetic core provided by manufacturers is limited and usually given not in the frequency of flux density of your design choice.

In the measurement setup[1][2][3|to construct core losses, it is necessary to amplify the AC signal to magnetize the core under test by a wide bandwidth power amplifier. The power required to magnetized the core increases with the volume of the core. Measurement using larger magnetic cores would therefore need a larger driving power to magnetize the core. On the other hand using smaller magnetic cores, the measurement of small signal has to be done accurately. In this case the accuracy of all equipment used becomes critical in the measuring process.

The purpose of this paper is to introduce simple measurement technique for constructing core loss of magnetic cores under sinusoidal high frequency excitation. The method is suitable for determining core-loss of lowloss type magnetic components. The power requirement for this measurement is small. The method provides reasonably accurate results as the determination of the core loss is no significantly affected by the parasitic of the simple measurement setup.

\section{MEASUREMENT OF THE MAGNETIC CORE LOSS}

The core loss in a magnetic material under AC magnetization consists of three components: the hysteresis loss, the eddy current loss, and the residue loss. The hysteresis loss and current loss contribute a significant portion of the core-loss. The method proposed determines the core loss by direct method. This is done by

measuring the voltage and current using an impedance analyzer, and the core loss is constructed from these measurements.

\subsection{Inductor equivalent circuit}

Consider an inductor with a magnetic core. The inductor is modelled as a complex impedance either as an equivalent series circuit or as an equivalent parallel circuit shown in Fig.1.

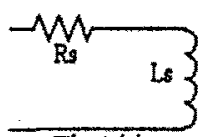

Fig 1(a)

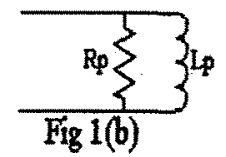

It is assumed that the flux in the magnetic core is not in saturation. As an equivalent series circuit, the impedance $Z_{s}$ is expressed as

$$
Z_{s}=R_{s}+j X_{s}
$$

where $R_{s}$ is the equivalent series resistance, and $X_{s}$ the equivalent series reactance. The phase angle $\theta$ is given as

$$
\theta=\tan ^{-1} \frac{X_{3}}{R_{s}}
$$

where

$$
X_{s}=\omega L_{s}
$$

$L_{s}$ is the equivalent inductance of the inductor winding. For parallel circuit, the impedance $Z_{s}$ is expressed as

$$
\frac{1}{Z_{s}}=\frac{1}{R_{p}}+\frac{1}{j X_{p}}
$$

$R_{p}$ is the equivalent parallel resistance, $X_{p}$ the equivalent reactance.

\subsection{Core Loss Measurement of Low-loss Magnetic Ma- terials}

For magnetic materials of low-loss and high permeability[1], the phase angle $\theta$ is near $90^{\circ}$. Since the loss power 
of the device under test is constructed from the measured amplitudes of voltage, current, and the phase angle $\theta$, the accuracy of the constructed power is largely dependent on the accuracy of the phase angle $\theta[1][4]$. Various direct methods reported in [4] indicated the significant errors in power loss measurement from extra phase shift associated with the parasitic in the measurement errors or delays in the measuring equipment.

\subsection{Determination of core-loss by resonant technique}

The problem mentioned in section 2.2 can be overcome if $\theta$ is effectively near or equal to zero. In this case the constructed power loss is not sensitive to measurement parasitic. At $\theta=0^{\circ}$ the circuit is in the resonant state. This state is implemented by connecting a suitable value capacitor $\mathrm{C}$ in series with the inductor winding. At the resonance or near resonance, the constructed power is no relation or least sensitive to phase errors. During resonance, the power from the source is effectively converted into core loss.

In order to maintain constant flux density, the induced voltage across the inductor winding should be maintained constant using the following relationship

$$
B_{m}=\frac{E}{4.44 f N A_{m}}
$$

where $B_{m}=$ maximum flux density $(T)$

$N=$ number of turns of the inductor winding

$A_{m}=$ cross sectional area of the magnetic core $\left(\mathrm{m}^{2}\right)$

$E=$ rms value of the induced voltage of the inductor winding $(V)$

$f=$ frequency $(\mathrm{Hz})$

At certain frequency $f, B_{m} \propto E$ and

$$
e(t)=i(t)\left(R_{s}+j \omega L_{s}\right)
$$

where

$e(t)=$ instantaneous voltage across the inductor wind$\operatorname{ing}(V)$

$i(t)=$ instantaneous current $(A)$

The rms value of current $I$ is given by

$$
I=\frac{E}{\sqrt{R_{s}^{2}+\left(\omega L_{s}\right)^{2}}}
$$

With series capacitance $C$, the voltage required becomes $E$

$$
e^{\prime}(t)=i(t)\left(R_{s}+R_{c a p}+j \omega L_{s}+\frac{1}{j \omega C}\right)
$$

where $R_{c a p}=$ equivalent resistance value of the capacitor, $e^{\prime}(t)$ is the instantaneous voltage with $C$ in series. Now the circuit current should be kept constant to maintain the same flux density, where

$$
I=\frac{E^{\prime}}{\sqrt{\left(R_{s}+R_{c a p}\right)^{2}+\left(\omega L_{s}-\frac{1}{\omega C}\right)^{2}}}
$$

Combining Eq.7 and Eq.9 gives

$$
E^{\prime}=\frac{\sqrt{\left(R_{s}+R_{\text {cap }}\right)^{2}+\left(\omega L_{s}-\frac{1}{\omega O}\right)^{2}}}{\sqrt{R_{s}^{2}+\left(\omega L_{s}\right)^{2}}} E
$$

At given operating frequency $f$, parameters $R_{s}$ and $L_{s}$ are measured by impedance analyzer. The required $C$ for resonance or near resonance is then determined. The driving voltage $E^{\prime}$ for the maintaining of certain flux density in the core is determined using Eq.10.

When resonance occurs,

$$
\omega L_{s}-\frac{1}{\omega C}=0
$$

The capacitance $C$ required is

$$
C=\frac{1}{\omega^{2} L_{0}}
$$

Eq.10 becomes

$$
E^{\prime}=\frac{R_{s}+R_{\text {cap }}}{\sqrt{R_{s}^{2}+\left(\omega L_{s}\right)^{2}}} E
$$

It can be deduced that $E^{\prime}$ is much smaller than $E$ for the same flux density since at high frequency $\left|\omega L_{s}\right| \gg$ $\left|R_{s}+R_{\text {cap }}\right|$. Then $E \cong \frac{\omega L_{s}}{R_{s}+R_{\text {cap }}} E^{\prime}$

In this paper, the determination of core-loss measurement is obtained from an experimental setup using the HP 4194A impedance analyzer(IA) and a PC for data processing. The setup is shown in Fig.2.

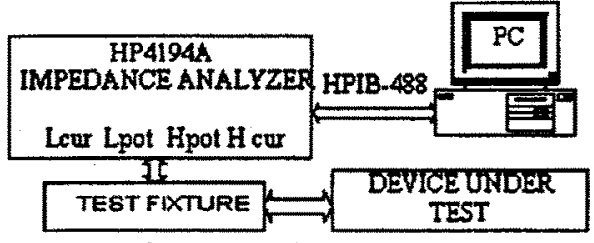

Fig 2 Experiment setup

From equivalent circuit of Fig.1a and Fig.1b,

$$
R_{p}=R_{s}+\frac{X_{s}^{2}}{R_{s}}
$$

At resonance,

$$
R_{p}=R_{s}
$$

The power loss per volume is calculated using the following formula:

$$
P_{e}=\left(\frac{E^{\prime}}{R_{s m}}\right)^{2} R_{s} / V_{e}
$$

where $V_{e}$ is the volume of the magnetic core. $R_{s m}=$ $R_{s}+R_{\text {cap }}$

\subsection{Measurement procedure}

The experiment procedure for constructing the core loss using the impedance analyzer is summarized as following.

(a) Set up IA functions such as OPEN and SHORT compensation and input flux density, frequency range, inductance factor, core type and size parameters by reading the data to the computer.

(b) Measure the inductance values at each frequency 
point and calculate the corresponding resonant capacitor values.

(c) Perform frequency sweep at the frequency range,ie adjust $f$ to get the resonant frequency $f_{0}$. To stabilize the flux in the magnetic core, the analyzer sweeps through the range of the frequency several times. The oscillation voltage level of the first frequency will be set based on the impedance value $X_{s}$ obtained when measuring the inductance before compensation at this frequency.

(d) Measure $E^{\prime}, R_{s m}, f_{0}$ at the resonant frequency.

(e) Compute the flux density $B_{m}$ automatically using Eqn.(5) and (13a). The flux density will hence be

$B_{m}=\frac{E}{4.44 N A_{m} f}=\frac{K L_{s} E^{\prime}}{N A_{m} R_{s m}}$

where $K=2 \pi / 4.44, R_{s m}=R_{s}+R_{c a p}$

(f) Compare $B_{m}$ with the required flux density B.

(g) Auto tune the $V_{s}$ to the required value.

(h) Record $E^{\prime}, R_{s m}, f$ as $B_{m}$ approaches or equal to B

(i) Increase the frequency to next step.

(j)Change the capacitor with the correct value.

(k) Repeat steps (c) to (j) for the rest of frequencies.

(l) Compute the loss $P_{e}$ at each point.

\subsection{Experiment Results}

The core sample under test is a toroid core,Tomita TRB632/2F1 with dimension of $6 \times 3 \times 2 \mathrm{~mm}^{3}$. The measurement was conducted at $10 \mathrm{mT}$ and $30 \mathrm{mT}$. Frequency range tested is $50 \mathrm{kHz}$ to $500 \mathrm{kHz}$. The result of core loss is shown in Fig 3. Table 1 shows the measured data for $\mathrm{B}=10 \mathrm{mT}$ only. From the results, it is observed that $E^{\prime}$ are much smaller than $E$ in accordance with Eq.13.

\section{CONCLUSION}

A simple experimental method for constructing core loss of high frequency low-loss and high permeability ferrite magnetic component is described. The equipment required only an impedance analyzer in the measurement setup. The resonant technique is introduced in the measurement process for two purposes. One is to reduce the errors of power measurement associated with directed-method measurement setup as mentioned in[1][2][3]. This is possible when the inductor test circuit is set at resonance to give a zero phase angle. The accuracy of this measurement depends only on a single equipment,namely the impedance analyzer. The second reason is to reduce the driving power required for magnetizing the core.

\section{REFERENCES}

[1]V.Joseph Thottuveli, Thomas G. Wilson, and Harry A.Owen, "High frequency measurement techniques for magnetic core", IEEE Trans on Power Electronics, Vol.5, no.1,January 1990. [2]David K.Conry, George F.Pierce," Measurement techniques for the design of high-frequency SMPS transformers",Proceedings of Virginia Power Electronics Center Seminar,1988.

[3]P.M.Gradzki and F.C.Lee,"High-Frequency core loss characterization technique based on impedance measurement",High Frequency Power Conference,1991,Toronto,Canada.

[4]F.Dong Tan, Jeff L. Vollin and Slobodan M.Cuk, " A practical Approach for magnetic Core-loss Characterization", IEEE APEC,1993.

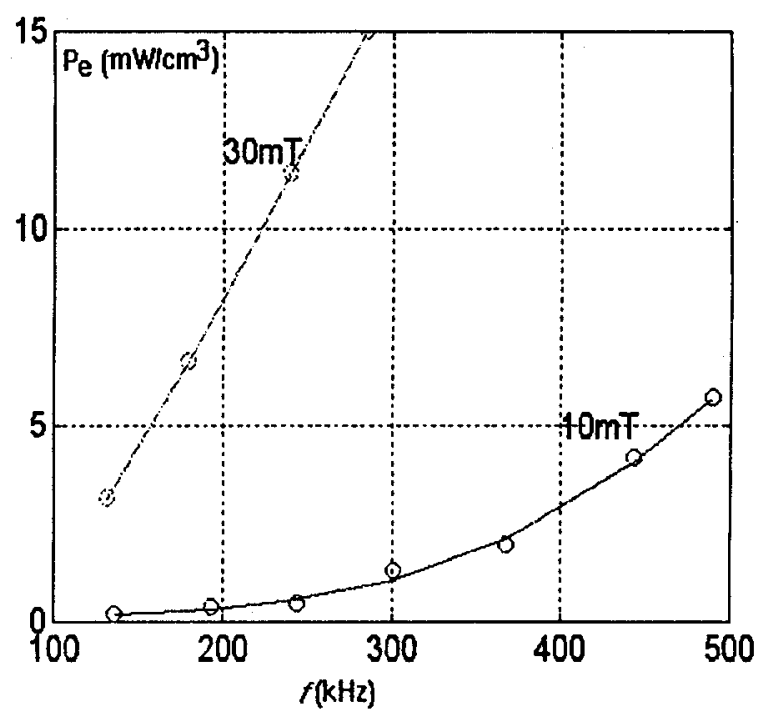

Fig 3 Core loss vs fiequency

Table 1

\begin{tabular}{|c|c|c|c|c|c|c|c|c|c|}
\hline $\mathrm{f}$ & $\mathrm{E}$ & $L_{s}$ & cap & $R_{c a p}$ & $R_{s m}$ & $R_{s}$ & $E^{\prime}$ & $\mathrm{P}$ & $P_{e}$ \\
\hline$(\mathrm{kHz})$ & $(\mathrm{V})$ & $(\mu \mathrm{H})$ & $(\mathrm{nf})$ & $(\mathrm{ohm})$ & $(\mathrm{ohm})$ & $(\mathrm{ohm})$ & $(\mathrm{V})$ & $(\mathrm{mW})$ & $\left(\mathrm{mW} / \mathrm{cm}^{3}\right)$ \\
\hline 136 & 0.097 & 48.58 & 28.17 & 1.14 & 2.19 & 1.05 & 0.0056 & 0.0066 & 0.1833 \\
\hline 194 & 0.141 & 47.00 & 14.32 & 1.45 & 3.50 & 2.05 & 0.0088 & 0.0128 & 0.3556 \\
\hline 245 & 0.182 & 50.17 & 8.41 & 3.52 & 5.78 & 2.56 & 0.0145 & 0.0160 & 0.4444 \\
\hline 301 & 0.228 & 50.01 & 5.59 & 2.00 & 9.30 & 7.30 & 0.0232 & 0.0456 & 1.2667 \\
\hline 368 & 0.288 & 50.01 & 3.74 & 3.58 & 14.84 & 11.26 & 0.037 & 0.0704 & 1.9556 \\
\hline 443 & 0.356 & 51.01 & 2.53 & 4.73 & 27.53 & 23.80 & 0.068 & 0.1488 & 4.1333 \\
\hline 490 & 0.396 & 50.72 & 2.08 & 4.18 & 36.84 & 32.66 & 0.091 & 0.2041 & 5.6694 \\
\hline
\end{tabular}

